\title{
Caracterización de las capacidades productivas en la población económica activa del barrio La Ceiba de Baranoa, Atlántico, 2018
}

\section{Characterization of productive capabilities in the active economic population of La Ceiba Baranoa Atlántico district, 2018}

\author{
Luis Fernando Escorcia-Márquez iD \\ Corporación Universitaria Minuto de Dios, Barranquilla, Colombia. \\ Johanna Orfelina Padilla-Ortiz iD \\ Corporación Universitaria Minuto de Dios, Barranquilla, Colombia \\ Elkyn Rafael Lugo-Arias \\ Corporación Universitaria Minuto de Dios, Barranquilla, Colombia. \\ Franklyn Ferrer-Manotas iD \\ Corporación Universitaria Americana, Barranquilla, Colombia.
}

\begin{abstract}
Resumen
Objetivo: caracterizar la capacidad productiva en la población económica activa y en situación de pobreza con estrato 1 bajo del barrio la Ceiba del municipio de Baranoa Atlántico, Colombia, para el año 2018. Método: Se utilizó el enfoque de la teoría de las capacidades productivas y las funciones institucionales del gobierno en las políticas públicas, mediante la aplicación de encuestas y entrevistas pre estructuradas, dirigidas a toda la población: 33 jefes de cada hogar y sus parientes en edad de trabajar se analizaron las dimensiones aspectos sociales como educativos, salud, capacidades físicas y de competencias; económicos, productivos, financieros, tecnológicos y comerciales. Resultados: Se encontró que las pocas personas en edad de trabajar, que han decido participar en las estrategias de formación y emprendimiento de su propio negocio, han logrado mejorar la situación económica, dado que han aumentado sus capacidades productivas y por ende el bienestar de sus hogares. Discusión: la falta de cobertura y de atención de las instituciones en proyectos productivos con las comunidades se da por la limitación del presupuesto. Conclusión: la mejora de las capacidades económicas conlleva a la prosperidad integral, y esto se deriva en gran parte de la generación de valores económicos, originalmente representado en términos financieros y económicos, los cuales son rentabilidad económica, progreso, innovación social y acumulación de conocimiento en la generación de sus propios recursos.
\end{abstract}

Palabras clave: capacidades, heterogeneidad, desarrollo económico, teorías sociales, desarrollo humano.

Clasificación JEL: I31, I32, D63 JEL: A14, I31, O10

\section{Autores de} Correspondencia

lescorciama@uniminuto.edu.co jpadillaort@uniminuto.edu.co elkyn.lugo@uniminuto.edu.co fferrer@coruniamericana.edu.co

Recibido: 06-05-19 Aceptado: 15-08-19

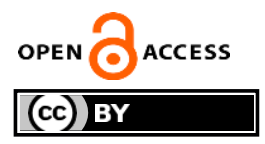

Copyright (c) 2019

Desarrollo Gerencial

\begin{abstract}
Objective: This paper aims to characterize the productive capacity in relation with active economic population taking into account a situation of poverty show in a low stratum (1) of La Ceiba neighborhood La Ceiba in the municipality of Baranoa Atlántico from Colombia, for 2018. Method: The productive capacities approach and the institutional functions of the government in public policies were used; surveys and pre-structured interviews aimed at the entire population were applied, as follows: 33 heads of each household and their relatives of working age, the dimensions of the social aspects are analyzed (educational, health, physical abilities and skills), economic, productive, financial, technological, commercial, among other aspects. Result: It was found that few people of working age, who have decided to participate in the training and entrepreneurship strategies of their own business, they have improved the economic situation of their homes, since they have increased their productive capacities $\mathrm{s}$ and your well-being. Discussion: The lack of coverage and attention in relation with productive projects with the communities by the institutions due to the limitation of their budget is discussed. Conclusion: The improvement of economic capabilities leads to its overall prosperity; this is where much of the generation of economic values, originally represented in financial and economic terms, derives: economic profitability, progress, social innovation and the accumulation of knowledge in the generation of its own resources.
\end{abstract}

Keywords: capacities, heterogeneity, economic development, social theories, human development. JEL Classification: I31, I32, D63, A14, I31, 010.

Como citar este artículo (Apa):

Escorcia-Márquez, L. Padilla-Ortiz, J., Lugo-Arias, E. \& Ferrer-Manotas, F. (2019). Caracterización de las capacidades productivas en la población económica activa del barrio La Ceiba de Baranoa, Atlántico, 2018. Desarrollo Gerencial, 11(2), 1-22. DOI: https://doi.org/10.17081/dege.11.2.3433 


\section{Introducción}

Este estudio surge gracias al compromiso y la responsabilidad social del grupo y semillero de investigación de la Corporación Universitaria Minuto de Dios, así como del Departamento de Proyección e Innovación Social, donde a través de un trabajo de campo se pudo detectar las necesidades y falta de condiciones con respecto a las oportunidades y equidad de género que contribuyen a aumentar las capacidades económicas de generación de ingresos en las familias vulnerables del barrio La Ceiba del municipio de Baranoa, Atlántico (Universidad Minuto de Dios, 2016).

Mediante esta investigación, se puede estructurar una propuesta o recomendación que mejore las elecciones de alternativas productivas para la población vulnerable, que habita en el barrio la Ceiba de Baranoa, Atlántico, con base a la caracterización o diagnóstico de las capacidades, toda vez que este proyecto buscará llamar la atención del gobierno, las empresas, las universidades, las fundaciones y demás organizaciones, para que trabajen en beneficio de esta población, dado que esta acción, permitirá que las familias obtengan una mayor remuneración económica y que sepan emprender y administrar sus finanzas.

De manera preliminar, se precisa la importancia de destacar que una de las mayores y principales características que sustenta la justificación de este proyecto, fue la identificación de los factores y elementos que se relacionan directamente con la situación de vulnerabilidad en la que conviven las comunidades menos favorecidas de estas zonas geográficas que padecen altas necesidades económicas, educativas, sociales y culturales. Pues a través del desarrollo de un exhaustivo proceso de observación que se efectuó, se logró establecer una serie de análisis riguroso que se tejieron con la finalidad de obtener un diagnóstico en profundidad, el cual se fraguó, además, a partir del hallazgo de información recopilada mediante los testimonios que se recogieron. Paralelo a ello se efectuó un inventario de las capacidades y funcionamiento asociados al acceso libre y equitativo: a la prestación de los servicios públicos, de salud, educativa, social, instituciones del gobierno, recursos, etc.

En la década de los noventas, todavía existía desigualdad y falta de la presencia del estado en las zonas rurales de Colombia, con esto la falta de oportunidades, empleo y NBI, trajo desigualdad, y pobreza, lo cual generó conflictos sociales y violencia entre grupos armados, acompañados de injusticia y violación de los derechos humanos. Esta situación obligó a la fuerza que las familias se desplazaran a las zonas marginadas del área metropolitana, cercana a Barranquilla, para buscar mejores condiciones de vida, y así lograr satisfacer sus necesidades básicas, de esta forma recuperar lo que dejaron o perdieron en sus tierras; dado las anteriores causas, se vieron obligados a invadir los terrenos que hoy formaron el barrio, lo cual generó el problema central de baja capacidad productiva en la formación y en la generación de ingresos, los cuales hacen que las familias se encuentran aún en condición de vulnerabilidad, en el barrio La Ceiba del municipio de Baranoa, Atlántico, estableciéndose en este, para intentar empezar una nueva vida con sus familias.

Las comunidades en este barrio, se encuentran en su mayoría, en situación de pobreza, por lo cual realizar esta propuesta permitirá conocer su diagnóstico socioeconómico, para proponer alternativas de mejoramiento de los ingresos y la calidad de vida de sus habitantes, mediante la identificación y establecimiento de estrategias y planes de acción adecuadas, los cuales generen, una cultura en cuanto a la creación de alternativas productivas, que capacite a 
Luis Escorcia-Márquez, Johanna Padilla-Ortiz, Elkyn Lugo-Arias, Franklyn Ferrer-Manotas

los habitantes de los barrios intervenidos y que sepan articularse en el mercado y comercializar sus productos, utilizando la gestión del conocimiento, la ciencia, la tecnología, la innovación social, para vender por medio de la internet y los aplicativos diseñados por el Ministerio de agricultura del Gobierno, tales como: Agronet, entre otros, de tal manera, aumenten sus clientes, pedidos, cargas, sus ingresos y puedan mejorar su calidad de vida y las de sus familias.

Es por esto, que las oportunidades en las actividades económicas existentes, actualmente, en este barrio, no revisten de una economía sólida y sostenible en las familias, puesto que esta es dinámica y variable, pero que sobre todo, presenta falencias en el componente de la capacidad intelectual, que tienen cada uno de ellos para llevarlas a cabo; y por tanto la cultura empresarial en emprendimiento empírico es totalmente nula, amenazante y peligrosa en toda su magnitud. Dada la situación anterior, se formula la siguiente pregunta de investigación:

¿Cuál es la situación de caracterización o el nivel de las capacidades productivas que presenta la población vulnerable económicamente activa del barrio la Ceiba de Baranoa, Atlántico?

En este sentido, este trabajo tuvo por objetivo caracterizar el nivel de la capacidad productiva de la población vulnerable, económicamente activa del barrio la Ceiba en el municipio de Baranoa, Atlántico, Colombia.

En la primera sección de este trabajo se muestra la fundamentación teórica de las capacidades económicas y funciones humanas, seguido del diseño metodológico, la caracterización de la situación social en educación, salud y capacidades productivas, económicas y de ciencia, tecnología e Innovación en las personas en edad de trabajar del barrio Baranoa. Por último, se derivan las conclusiones y las discusiones de los resultados de la investigación.

\section{Fundamentación teórica}

A nivel internacional existen varios estudios que explican la situación socioeconómica de la problemática de pobreza en diferentes países, y sus regiones. Entre los cuales se destacan los siguientes:

\section{Teoría de las capacidades productivas y las funciones.}

Sen (1995), realizó un estudio para reducir la pobreza en la India, aplicando la teoría de las capacidades y las funciones de las agencias o instituciones, la cual se aplicó arrojando excelentes resultados que permitieron la participación justa y equitativa de todas los géneros en los proyectos sociales de interés común, el acceso a los espacios libres, entre otras oportunidades de áreas estratégicas, las cuales tuvieron un alto impacto en el mediano y largo plazo, permitiéndole obtener un premio nobel en economía, dado que su planteamiento generó una acumulación en el desarrollo humano, que provocó una estabilidad en la ampliación de la generación de ingresos o recursos de la mayoría de las familias participantes (Evans, 2002; Córdoba, 2006; Walker \& Unterhalter, 2007; Stiglitz et al. 2010; Nussbaum, 2011; Wells, 2012; Hatakka, 2013; Urquijo, 2014; Michalos, 2017; Dworkin, 2018).

La libertad de la agencia: esta se ve afectada por tres factores de conversión: características personales como metabolismo, condición física, sexo, habilidades de lectura, inteligencia; características sociales como políticas públicas, normas sociales, prácticas discriminatorias, roles de género, jerarquías sociales, relaciones de poder y características ambientales como clima, infraestructura, instituciones, bienes públicos (Robeyns 2005). 
Al aceptar el papel de los factores de conversión que afectan el proceso de realizar las cosas, el enfoque de capacidad incluye elementos sociales y estructurales en el proceso evaluativo. Sin embargo, muchos autores han criticado los escritos de Sen (1995), por estar demasiado enfocado en el individuo. No obstante, Sen (2018) argumenta, que el enfoque de capacidad no puede ser etiquetado como excesivamente individualista de ninguna manera simple, ya que, al igual que factores de agencia y conversión, identifica las funciones sociales como participar en la vida de la comunidad, comunicar, estar bien integrado en la sociedad y usando el famoso ejemplo de Adam Smith, aparecer en público sin vergüenza.

Capacidad, apertura e incompletitud: si bien no acepta explícitamente el concepto de capacidades colectivas, Sen (1992) sostiene que el enfoque de capacidades es deliberadamente incompleto, ya que no especifica una lista de capacidades o funciones valiosas. Además, no proporciona pautas prácticas claras a los profesionales o investigadores sobre cómo evaluar o identificar capacidades. Robeyns (2005) critica este carácter incompleto al afirmar que la amplitud y la naturaleza multidimensional y dependiente del contexto del enfoque, le impiden tener una importancia práctica y operacional. Tales críticas llevaron a los demás teóricos a proponer una lista de capacidades que buscaban operacionalizar el enfoque de Sen.

Adaptación y aplicación del enfoque de capacidad: La revisión anterior del enfoque de capacidad indica algunos de sus componentes y conceptos que deberían adaptarse para elaborar un enfoque para el desarrollo y convertirse en una alternativa de desarrollo radical, el enfoque de capacidad debe abordar los procesos locales y estructurales; el marco conceptual necesita componentes claros que puedan ser utilizados por los profesionales del desarrollo, mientras permanezcan abiertos y no impongan valores universales; y la participación y la información cualitativa deben incorporarse en el proceso de elaboración o evaluación de políticas y proyectos (Sen, 2018).

Para abordar tales postulaciones, este artículo propone: (a) un enfoque en los factores de conversión, transformando los recursos en funcionalidades logradas; (b) la incorporación del análisis de las relaciones de poder en dichos procesos; y (c) mecanismos para incluir métodos participativos en la aplicación del enfoque de capacidad.

De las capacidades a un espacio de capacidades: las aplicaciones del enfoque de capacidades hasta ahora se han centrado principalmente en el listado de capacidades y en evaluar el impacto de las políticas sobre ellas. Este enfoque fue motivado por el deseo de enumerar las dimensiones concretas del bienestar para generar indicadores tangibles contra los cuales examinar las libertades de las personas. Sin embargo, al centrarse en los indicadores, los elementos del proceso que afectan la libertad de las personas, como las condiciones sociales y políticas subyacentes, no están claramente articulados (Sen, 2018).

El hecho de no desempaquetar el concepto de capacidad, lleva a los debates no resueltos sobre las capacidades colectivas frente a las individuales y las capacidades universales frente a las locales. Además, el enfoque de capacidad se mantiene a un nivel de abstracción de políticas y proyectos que dificulta que los profesionales apliquen y evalúen el valor que agrega al diseño y evaluación de proyectos (Sen, 2018). 
Esta teoría propone una perspectiva ligeramente diferente sobre el enfoque de capacidad, centrándose en los recursos y su transformación en funcionalidades logradas. Estos recursos pueden ser tangibles como escuelas, transporte y viviendas o intangibles como políticas. La transformación de los recursos se ve afectada por una serie de factores de conversión que varían de un contexto a otro, de persona a persona (Sen, 2018). En lugar de centrarse en las capacidades, esta aplicación se centra en el espacio de capacidades, que incluye la elección, la capacidad y la oportunidad de las personas para transformar los recursos en funcionalidades logradas.

Dentro del espacio de capacidad se incluyen factores individuales, locales y estructurales. Los factores individuales están asociados con las capacidades individuales, que pueden ser las condiciones físicas, los niveles de alfabetización, entre otros, por ejemplo, la ceguera y el analfabetismo son factores individuales que influyen en la capacidad de una persona para transformar los periódicos impresos en una mayor conciencia (Sen, 2018). Los factores locales pueden asociarse con instalaciones y normas colectivas, por ejemplo, la provisión de un campo de fútbol en un vecindario no significa que los niños locales tengan más espacio para el ocio, ya que los adultos podrían controlar el uso del campo y no permitir que los niños jueguen allí.

Por último, pero no menos importante, están los factores estructurales que configuran el espacio de capacidad. Los mecanismos de mercado y la estructura política son ejemplos de algunos de los procesos estructurales subyacentes, que afectan las libertades de las personas. Por lo tanto, en la aplicación del enfoque de capacidad para evaluar el bienestar de los pequeños productores de leche en Uganda, por ejemplo, es necesario tener en cuenta el impacto de los subsidios otorgados a los productores de leche en el mundo industrializado. Si la regularización de la tierra de los asentamientos informales en los países en desarrollo es el recurso evaluado a través del enfoque de capacidad, los factores estructurales se relacionan con las fuerzas formales del mercado que ingresan a dichos asentamientos debido a la regularización, expulsando a los más pobres al elevar el precio de la propiedad. El propósito de resaltar estos tres niveles de factores de conversión, es aclarar los diversos problemas que comprenden el espacio de capacidad. Estos tres niveles están interconectados, moldeando e influyendo entre sí (Sen, 2018).

\section{Teoría del microcrédito como alternativa de solución de la pobreza.}

El premio nobel, Yunus et al. (2006) decidió fundamentar esta teoría, dada las duras condiciones de trabajo e ingresos, que observó en otras personas, especialmente en las mujeres, que se encargaban de las etapas posteriores a la cosecha, desde la limpieza hasta el empaque y la comercialización de los productos. A Yunus et al. (2006) le resultó evidente que la pobreza era mucho más dura entre la población rural sin tierra, constituida masivamente por jefas de hogar.

De esta manera, cualquier esfuerzo por combatir la pobreza en las áreas rurales requería, entonces, involucrar a los trabajadores sin recursos económicos, en especial a las mujeres. La situación le permitió identificar que la pobreza de quienes no poseen tierras está asociada, fundamentalmente, al hecho de no disponer de cantidades ínfimas de dinero para invertir en capital de trabajo, ni poder acceder a mecanismos de endeudamiento. Ciertamente, sin acceso al sistema bancario moderno, la única alternativa que tenían estas personas era recurrir a los prestamistas de la zona, que entre otras características cobraban exorbitantes tasas de interés (Yunus, et al. 2006). 
Buscando soluciones que fueran más allá del nivel personal o de una intervención por una sola vez, Yunus empezó a explorar posibilidades de establecer una salida institucional. La aceptación del crédito, el rápido repago y la continua demanda de préstamos adicionales por parte de los productores mostraba la necesidad de generar mecanismos más institucionales, masivos y permanentes. Así lo entendió Yunus y así surgió la motivación inicial para crear lo que ha llegado a ser el Grameen Bank (Yunus et al. 2006).

En este punto también empezaron los grandes desafíos y enfrentamientos, que Yunus sostuvo con la banca comercial y las instancias gubernamentales, a las que trató de convencer de que valía la pena prestarles a los pobres. Diversas anécdotas que posteriormente ha narrado, le mostraron la resistencia de estas instituciones para adaptarse a esta faceta de la realidad y otorgar créditos de este tipo, aun a tasas de interés de mercado. Yunus incluso se involucró avalando personalmente préstamos bancarios para algunos microproductores, sin que el éxito en el repago convencieran a los bancos de expandir sus actividades. Además de sostener diversos mitos sobre la actividad económica y la disposición a pagar de los pobres, los banqueros tenían sus propios principios para hacer negocios, al parecer incompatibles a priori con las características de estos clientes potenciales (Yunus, et al. 2006).

En este aspecto, Yunus et al. (2006), decidieron crear un banco dedicado a los pobres. Luego de una suerte de circunstancias favorables y gracias al reconocimiento personal que él había ganado, tanto en el medio bancario como en la opinión pública, el Grameen Bank inició sus operaciones como una filial experimental del estatal Banco Agrícola de Bangladesh (BKB). Ello le permitió incluso denominar a su filial Grameen, para reflejar su orientación rural, más allá de actividades meramente agrícolas, lo que le facilitaría la atención a los clientes rurales pobres.

Asimismo, Petrucci, Salvati y Seghieri (2003), utilizaron no sólo los mapas de pobreza sino también los modelos de regresión espacial, con el fin de explorar la dimensión geográfica de la pobreza en Ecuador y su posible vínculo con las condiciones ambientales. En este estudio encontraron correlación espacial significativa en las medidas de pobreza utilizadas, y destacaron que no tener en cuenta indicadores geográficos en la medición de la pobreza, puede llevar a que las medidas utilizadas sean engañosas y que no reflejen la verdadera situación de la población.

Uno de los más recientes trabajos, en donde se explora la relevancia del uso de mapas de pobreza y seguridad alimentaria, como instrumento de análisis, diseño de política e implementación en los sectores rurales de los países en vía de desarrollo, es el de Davis (2003), en el cual utiliza diferentes metodologías de análisis de los tipos de pobreza, con el fin de determinar su relevancia en la aplicación de diferentes políticas. Indican además que la escogencia adecuada de una u otra metodología depende, principalmente, de factores como el objetivo del análisis, criterio de definición de la pobreza, limitaciones de información y costo.

Otros estudios, como el de Ospina y Giraldo (2005), revisaron las principales metodologías para evaluar y analizar los temas de la pobreza y la distribución del ingreso, las teorías, y las metodologías asociadas a ellas, les permitieron realizar los análisis de desigualdad socio económica, clasificando la población conforme al segmento social al que pertenece. La caracterización de estas categorías, permitió diseñar las políticas sociales y medir su incidencia en el desarrollo y crecimiento económico de un país.

Por otra parte, en Colombia, se destacan estudios sobre los factores económicos determinantes de la pobreza como los de Sánchez y Núñez (1999), quien en su trabajo sobre los determinantes de la pobreza urbana, utilizaron 
Luis Escorcia-Márquez, Johanna Padilla-Ortiz, Elkyn Lugo-Arias, Franklyn Ferrer-Manotas

variables de tipo demográfico, macroeconómico y de cambio estructural. La principal conclusión es que en el largo plazo variables como el desempleo, el tipo de cambio depreciado y el deterioro de los términos de intercambio, están relacionados con los mayores niveles de pobreza. Por otro lado, mayores niveles de escolaridad y mayor productividad multifactorial y laboral se encuentran asociados con menores niveles de pobreza en la población.

De la misma manera, Pérez (2005), realizó un análisis espacial y estimó los indicadores de las necesidades básicas insatisfechas (NBI) y la calidad de vida (CV), de la pobreza en Colombia; en el cual buscaba establecer si existían evidencias de dependencias en las medidas de la pobreza de los departamentos y municipios en Colombia, a través de los Cálculos I Morgan, los cuales arrojaron como resultados evidencias significativas de la dependencia espacial municipal como departamental, determinando que la ubicación geográfica incide en la pobreza del país. Es decir, la mayoría de los municipios pobres están rodeados de otros municipios pobres, y los municipios ricos rodeados de municipios ricos, estos factores naturales, externalidades o combinación de ambas ayudan a este fenómeno de la pobreza siga en aumento.

En otro estudio más reciente realizado por Galvis y Meisel (2012), en el Banco de la Republica sobre los factores determinantes de la pobreza en Colombia, utilizaron un método de análisis económico espacial, donde encontraron como resultado en la distribución de la pobreza, que los departamentos del Choco tiene un rezago en el ingreso de más de dos siglos, frente a Bogotá, así mismo, como otros departamentos, que tienen mayores niveles de pobreza.

\section{Método}

\section{Diseño.}

Con base en el marco teórico en el que se soporta esta investigación y los objetivos que se plantearon, se procedió al desarrollo de estos últimos mediante un estudio descriptivo de las características que identifican el contexto desde un enfoque mixto, mediante datos cuantitativos y cualitativos de fuente primaria con la participación activa de las familias focalizadas en el barrio La Ceiba del municipio de Baranoa. Este barrio cuenta con 33 familias y se encuentra ubicado en el perímetro urbano del municipio de Baranoa-departamento del Atlántico sectorizados en estrato 1.

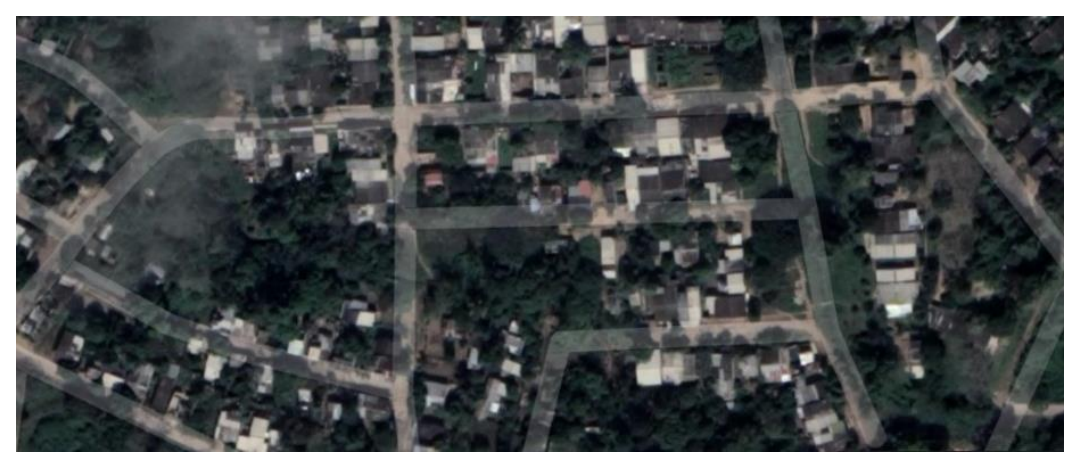

Figura 1. Ubicación del Barrio la Ceiba, municipio de Baranoa-Atlantico. Nota. Fuente: Google mapas (2018) 
Asimismo, se emplearon criterios teóricos y técnicos, indicadores de capacidades económicas y humanas con las dimensiones de las condiciones sociales de acceso al conocimiento, a la educación, a la formación, a la salud, a la tecnología, la generación de ingresos, la participación igualitaria de género en la población, según la teoría de Amartya Sen, relacionados al de estudio de la pobreza como un problema o fenómeno económico y social, adecuados para el análisis de los datos, mediante instrumentos, herramientas y argumentos necesarios para caracterizar las capacidades y funciones humanas en las familias en situación de pobreza de las poblaciones asentadas en el barrio de estudio.

\section{Participantes.}

En este proceso se efectuaron preliminarmente entrevistas a un grupo de habitantes en edad de trabajar, entre hombres, mujeres, jóvenes y adultos mayores, que narraron la realidad social, económica, financiera, comercial y de tecnología, dentro de las cuales caracterizaron la actual actividad a la que se dedican desde sus inicios, en todos los ámbitos a nivel social, cultural, económico, político, tecnológico, entre otros. Aquí, se ahondó en la búsqueda por entrelazar cada uno de esos relatos que se recopilaron, el sentir de los personajes involucrados con la reflexión del investigador y la producción conjunta de conocimiento, los cuales, forjaron la necesidad fundamental de sintonizar esta propuesta.

Además, se emplearon la encuesta y la entrevista no estructurada, con el propósito de que las personas a las que se acudió para conocer su información estadística descriptiva frente al tema, tales como personas en edad de trabajar, tanto hombres como mujeres con una edad mayor de 18 años, con la finalidad de que expresaran sus respuestas con total libertad y autónoma de dar su opinión, de tal manera que proporcionen la información requerida de la situación actual.

Como fuentes primarias para la recolección de la información, se utiliza la encuesta, en una población de 33 jefes de los 33 hogares existentes ( $n=N=33$ hogares) en el barrio la Ceiba de Baranoa, así como a las personas en edad de trabajar, tomando toda la población para caracterizar las capacidades económicas del barrio la Ceiba de Baranoa, a las cuales se les realizará su formulación y tabulación.

\section{Instrumentos de recolección de información y procedimiento.}

Se empleó la utilización de la encuesta y la entrevista estructurada, con el propósito de que las personas dieran a conocer su situación y su posición frente al tema. Este proceso tuvo como objetivo corroborar algunos de los aspectos obtenidos a partir de las entrevistas y cuestionarios sobre algunos de los aspectos sociales, económicos y productivos, obtenidos a partir de las entrevistas y cuestionarios, realizadas con evidencias, usando: papel, lápiz, cámara fotográfica, grabadora digital de voz y cámara de video.

\section{Resultados}

En esta sección se presentarán los resultados de la caracterización de las capacidades productivas en la población objeto de estudio, en la cual teniendo en cuenta el instrumento desarrollado según la teoría de las capacidades de Sen (1995), se realizó en primera instancia, el diagnostico de las capacidades sociales; seguidamente se estimaron las 
Luis Escorcia-Márquez, Johanna Padilla-Ortiz, Elkyn Lugo-Arias, Franklyn Ferrer-Manotas

capacidades productivas; luego las capacidades económicas/financieras; y por último, se diagnosticaron las capacidades tecnológicas de la información y la comunicación TIC's.

Las capacidades económicas, productivas y emprendedoras, que se identificaron y midieron para caracterizar a la población en edad de trabajar del barrio en estudio y que se encuentran en situación de pobreza fueron: educación, afiliación en salud, discapacidad para trabajar, ocupación, activos, ahorro, ingresos, subsidios, negocios, empleo y préstamos.

Así, de esta manera se identificó que la mayoría de los micro negocios encontrados, no cumplen con los normativas legales aceptadas, por ende la misión es realizar una propuesta con información de las buenas prácticas en los procesos productivos, para que se capaciten con esta, de tal forma que puedan conocer, aprender y emprender negocios sostenibles con potencial de crecimiento, y su legalización, llevando a establecer la contabilidad como unas de las herramientas principales del negocio y como resultado de un correcto desempeño económico (Landázury, et al. 2017; Lugo, Sáenz \& Lugo, 2018; Lugo \& Lugo, 2018; Lugo, et al. 2018; Landázury, et al. 2018 y Henríquez, et al. 2018).

Al implementar las alternativas productivas en esta comunidad, la economía comienza a beneficiar a toda la comunidad en general, a cambiar la calidad de vida personal, familiar, social y ambiental, preparándolos a través del conocimiento, a los diversos cambios que la sociedad vivencia continuamente, llevando a las localidades a un nivel socio-cultural y socio-económico estable y en avance.

\section{Caracterización de la situación social en educación, salud y capacidades productivas.}

\begin{tabular}{ccc}
\multicolumn{3}{c}{$\begin{array}{c}\text { Tabla } 1 . \\
\text { Sexo }\end{array}$} \\
\hline Sexo & $N^{\circ}$ de personas en edad de trabajar & Part. (\%) \\
\hline Masculino & 71 & $53.79 \%$ \\
\hline Femenino & 61 & $46.21 \%$ \\
\hline Total & 132 & $100.00 \%$ \\
\hline
\end{tabular}

En la tabla 1, se puede apreciar que 132 personas en edad de trabajar, de esta población económicamente activa, de los 33 hogares encuestados, el $53.79 \%$ es de género masculino y el $46.21 \%$ es femenino.

Tabla 2.

Estado civil

\begin{tabular}{ccc}
\hline Estado Civil & $N^{\circ}$ de personas en edad de trabajar & Part. (\%) \\
\hline Separado & 6 & $4.55 \%$ \\
\hline Soltero & 58 & $43.94 \%$ \\
\hline Casado & 29 & $21.97 \%$ \\
\hline Unión Libre & 39 & $29.55 \%$ \\
Viudo & 0 & $0.00 \%$ \\
\hline Total & 132 & $100.00 \%$ \\
\hline
\end{tabular}

Fuente: Elaboración propia mediante encuesta (2018). 
En la tabla 2, se observa que el $43.94 \%$ de la población económicamente activa se encuentran en estado civil solteros (58 personas), lo cual es alto y se puede explicar por la falta de ingresos para sostener un matrimonio y familia, también porque hay familias donde los padres niegan su estado civil de compromiso y porque se separan por problemas intrafamiliares o psicológicos. Así mismo, el 29.55\% viven en unión libre, el $21.97 \%$ está casado y ninguno esta viudo.

Tabla 3.

Conformación familiar

\begin{tabular}{ccc}
\hline Conformación del grupo familiar & $\mathrm{N}^{\circ}$ de personas en edad de trabajar & Part. (\%) \\
\hline Jefe de familia & 33 & $25.00 \%$ \\
\hline Conyugue & 25 & $18.94 \%$ \\
\hline Hermano(a) & 13 & $9.85 \%$ \\
\hline Padre/madre & 26 & $19.70 \%$ \\
\hline Hijo(a) & 35 & $26.52 \%$ \\
\hline Total & 132 & $100.00 \%$ \\
\hline
\end{tabular}

Fuente: Elaboración propia mediante encuesta (2018).

En la tabla 3, se observa que 35 personas ejercen el rol de hijos, 33 de jefes de familia, 25 de conyugues, 13 de hermanos y 26 entre padres y madres de familia.

Tabla 4.

Ocupación

\begin{tabular}{ccc}
\hline Ocupación & $\mathrm{N}^{\circ}$ de personas en edad de trabajar & Part. (\%) \\
\hline Independiente & 15 & $11.36 \%$ \\
\hline Jubilado & 5 & $3.79 \%$ \\
\hline Ama de casa & 24 & $18.18 \%$ \\
\hline Empleado & 35 & $26.52 \%$ \\
\hline Desempleado & 20 & $15.15 \%$ \\
\hline Estudiante & 33 & $25.00 \%$ \\
\hline Total & 132 & $100.00 \%$ \\
\hline
\end{tabular}

Fuente: Elaboración propia mediante encuesta (2018).

En la tabla 4, se puede observar que de las 132 personas en edad de trabajar, sólo el 11.36\% trabajan de manera independiente y sólo el $26.52 \%$ trabaja como empleado. Por su parte, los jubilados, son solamente el 3.79\% de las personas en edad de trabajar, el resto de las personas (59\%) se desempeña como estudiante, ama de casa y desempleado, esta última ocupación se considera alta con un 15.15\%, por encima del promedio departamental y nacional, según los niveles manejados en las cifras estadísticas del Dane.

La participación más alta fue la de ama de casa y estudiante, cada una por encima del $18.18 \%$. De la anterior situación, se refleja que los estudiantes jóvenes y solteros, son los que más se capacitan, se dedican más al estudio, mientras que la mujer ama de casa poco se capacita y se dedica al cuidado de sus hijos, esposo u otro familiar, porque depende de la ayuda del jefe de hogar. 
Tabla 5.

Nivel de escolaridad

\begin{tabular}{|ccc|}
\hline Nivel de escolaridad & $N^{\circ}$ de personas en edad de trabajar & Part. (\%) \\
\hline Secundaria completa & 37 & $28.03 \%$ \\
\hline Secundaria incompleta & 36 & $27.27 \%$ \\
\hline Primaria completa & 13 & $9.85 \%$ \\
\hline Universitaria & 16 & $12.12 \%$ \\
\hline Técnica o tecnológica & 15 & $11.36 \%$ \\
\hline Lee y escribe & 9 & $6.82 \%$ \\
\hline Analfabeta & 6 & $4.55 \%$ \\
\hline Total & 132 & $100.00 \%$ \\
\hline
\end{tabular}

Fuente: Elaboración propia mediante encuesta (2018).

En la tabla 5, se puede apreciar que más del 28\% de los habitantes han estudiado la secundaría completa, el $27.27 \%$ secundaría incompleta, en la educación profesional universitaria sólo el $12.12 \%$ ha estudiado En Instituciones de Educación Superior, el $11.36 \%$ es técnico o tecnólogo y el 9.885\% estudió la primaria completa. Por otro lado, el $11.37 \%$, no ha estudiado ni siquiera la primaria, el $4.55 \%$ son analfabetas y el $6.82 \%$ al menos, aprendió a leer y escribir por su propia cuenta.

Tabla 6.

Tipo de afiliación en salud

\begin{tabular}{ccc}
\hline Tipo de afiliación en salud & $N^{\circ}$ de personas en edad de trabajar & Part. (\%) \\
\hline Contributivo & 32 & $24.24 \%$ \\
\hline Subsidiado & 100 & $75.76 \%$ \\
\hline Total & 132 & $100.00 \%$ \\
\hline
\end{tabular}

Fuente: Elaboración propia mediante encuesta (2018).

En la tabla 6, el $75.76 \%$ de las personas en edad de trabajar, está afiliado en el régimen de salud subsidiado (estrato 1), mientras que el $24.24 \%$ se encuentra vinculado al régimen contributivo.

Tabla 7.

Tipo de discapacidad

\begin{tabular}{ccc}
\hline Discapacidad & $N^{\circ}$ de personas en edad de trabajar & Part. (\%) \\
\hline Visual & 2 & $1.52 \%$ \\
\hline Motora & 5 & $3.79 \%$ \\
\hline Del habla & 3 & $2.27 \%$ \\
\hline Mental & 1 & $0.76 \%$ \\
\hline Auditiva & 2 & $1.52 \%$ \\
\hline Ninguna & 119 & $90.15 \%$ \\
\hline Total & 132 & $100.00 \%$ \\
\hline
\end{tabular}

En la tabla 7, la población en edad de trabajar, en su mayoría el $90.15 \%$, no tiene ningún problema de discapacidad que le impida realizar una actividad productiva, sin embargo, el 3.79\% tiene limitaciones motoras, el $0.76 \%$ limitaciones mentales, el $2.27 \%$, presenta limitaciones en el habla y el $1.52 \%$ en la visión y audición. 
Tabla 8.

Nivel educativo del jefe de hogar

\begin{tabular}{ccc}
\hline Nivel educativo & Jefe de hogar & Part. (\%) \\
\hline Primaria & 6 & $18.18 \%$ \\
\hline Bachiller & 19 & $57.58 \%$ \\
\hline Técnico & 6 & $18.18 \%$ \\
\hline Pregrado & 0 & $0.00 \%$ \\
\hline Ninguno & 2 & $6.06 \%$ \\
\hline Total & 33 & $100.00 \%$ \\
\hline
\end{tabular}

Fuente: Elaboración propia mediante encuesta (2018).

En la tabla 8, se observa que ningún jefe de hogar tiene un nivel de educación alto o profesional, solo un 18.18\% es técnico, un $57.58 \%$ es bachiller y un $18.18 \%$ estudió hasta la primaria, sin embargo, es alta, la tasa de analfabetismo, la cual es del $6.06 \%$ del total de encuestados.

Tabla 9.

Percepción de la necesidad de capacitarse

\begin{tabular}{c|c|c|}
\hline ¿Considera que necesita capacitarse? & Jefe de hogar & Part. (\%) \\
\hline Si & 27 & $81.82 \%$ \\
\hline No & 6 & $18.18 \%$ \\
\hline Total & 33 & $100.00 \%$ \\
\hline
\end{tabular}

Fuente: Elaboración propia mediante encuesta (2018).

Según la tabla 9, el $81.82 \%$ de los jefes de hogar consideran que necesitan capacitarse y están dispuestos a prepararse, mientras que el $18.18 \%$ no tiene el deseo de hacerlo.

Tabla 10.

Beneficios de los programas de capacitación del Estado

\begin{tabular}{cccc} 
¿Ha sido beneficiario de los programas de capacitación del estado? & Jefe de hogar & Part. (\%) \\
Si & 9 & $27.27 \%$ \\
No & 24 & $72.73 \%$ \\
\hline Total & 33 & $100.00 \%$
\end{tabular}

Fuente: Elaboración propia mediante encuesta (2018).

Según la tabla 10, el 72.73\% de los jefes de hogar, no reciben ningún beneficio de los programas de capacitación del Estado, mientras que el $27.27 \%$, si las ha recibido.

Tabla 11.

Percepción en la elección de las áreas de capacitación

$\begin{array}{ccc}\text { Áreas en la que le gustaría capacitarse } & \text { Jefe de Hogar } & \text { Part. (\%) } \\ \text { Ventas (comercial) } & 14 & 42.42 \% \\ \text { Salud } & 0 & 0.00 \% \\ \text { Agropecuario } & 0 & 0.00 \% \\ \text { Administración de negocios } & 8 & 24.24 \% \\ \text { Industrial } & 2 & 6.06 \% \\ \text { Educación } & 6 & 18.18 \% \\ \text { Belleza } & 3 & 9.09 \% \\ \text { Total } & 33 & 100.00 \%\end{array}$

Fuente: Elaboración propia mediante encuesta (2018). 
Según la tabla 11, la mayoría de los 33 jefes de hogar encuestados, les gusta capacitarse y están interesados en estudiar: $42.42 \%$ en ventas, $24.24 \%$ en administración de negocios, $9.09 \%$ en belleza, $18.18 \%$ en educación, $6.06 \%$ en lo industrial y $0 \%$ en las áreas relacionadas con los sectores agropecuario y salud.

Tabla 12.

Percepción de las condiciones de vida del jefe de hogar ¿Cómo cree que serán las condiciones de vida de usted y su familia Jefe de Hogar Part. (\%) en un año?

\begin{tabular}{c|c|c|}
\hline Seguirán siendo iguales & 22 & $66.67 \%$ \\
\hline Mejoraran & 9 & $27.27 \%$ \\
\hline Empeoraran & 2 & $6.06 \%$ \\
\hline Total & 33 & $100.00 \%$ \\
\hline
\end{tabular}

Fuente: Elaboración propia mediante encuesta (2018).

Según la tabla 12, sólo 9 de los jefes de hogar (27.27\%), se encuentran optimistas de que su calidad de vida y bienestar de su familia, como va, mejorará más, el próximo año, el $66.67 \%$ piensa que seguirá igual y sólo el $6.06 \%$ piensa que empeorará. Estos últimos necesitan motivación y mayores capacidades productivas para mejorar sus condiciones de vida.

\section{Caracterización de la situación económica y financiera de las familias.}

Tabla 13.

Tipo de generación de ingresos del jefe de hogar

\begin{tabular}{ccc}
\hline ¿De qué manera se generan los ingresos de esta familia & Jefe de Hogar & Part. (\%) \\
\hline Trabajo formal & 16 & $48.48 \%$ \\
\hline Independiente & 7 & $21.21 \%$ \\
\hline Aporte familiar & 1 & $3.03 \%$ \\
\hline Subsidio & 4 & $12.12 \%$ \\
\hline Pensión & 5 & $15.15 \%$ \\
\hline Total & 33 & $100.00 \%$ \\
\hline Fuente: Elaboración propia mediante encuesta (2018).
\end{tabular}

Según la tabla 13, la mayoría de los jefes de hogar (48.48\%), generan los ingresos con su trabajo formal en empresas, el $21.21 \%$ lo genera con su trabajo independiente, el $12.12 \%$ lo genera por subsidios, el $15.15 \%$ por pensión y el 3.03\% por ayuda de familiares. Esto muestra que todos requieren mejorar sus ingresos y generar más ingresos, por medio del fortalecimiento de las capacidades productivas y el emprendimiento.

Tabla 14.

Subsidios de los miembros del grupo familiar ¿Ha recibido o recibe alguno de los siguientes subsidios?

Jóvenes en acción

Adulto mayor

Familias en acción

Desplazados

Red unidos

ONG/s

Iglesia

Total

Fuente: Elaboración propia mediante encuesta (2018).

\begin{tabular}{cc} 
Miembros & Part. (\%) \\
0 & $0.00 \%$ \\
1 & $25.00 \%$ \\
3 & $75.00 \%$ \\
0 & $0.00 \%$ \\
0 & $0.00 \%$ \\
0 & $0.00 \%$ \\
0 & $0.00 \%$ \\
4 & $100.00 \%$ \\
\hline
\end{tabular}

Fuente: Elaboración propia mediante encuesta (2018). 
La tabla 14, muestra las respuestas dadas en cuanto a los subsidios que brinda el estado; el jefe de hogar respondió que en su núcleo familiar, solamente 4 personas, reciben subsidios, siendo principalmente: $75 \%$ de Familias en acción (padres de niños) y $25 \%$ adulto mayor.

Tabla 15.

Trabajo lucrativo o negocio económico en la vivienda ¿En su vivienda se realiza algún trabajo o negocio que genere Jefe de hogar Part. (\%) ingresos económicos?

\begin{tabular}{cccc}
\hline Si & 19 & $57.58 \%$ \\
\hline No & 14 & $42.42 \%$ \\
\hline Total & 33 & $100.00 \%$ \\
\hline
\end{tabular}

Fuente: Elaboración propia mediante encuesta (2018).

Según la tabla 15, la mayoría de los jefes de hogar (57.58\%), si realizan negocios en sus casas, lo que les generan un ingreso adicional, mientras que el $42.42 \%$, no realiza ningún tipo de negocios, esto refleja que los jefes de hogar y sus familias, necesitan motivación y mayores capacidades productivas para mejorar sus condiciones de vida.

Tabla 16.

Ingresos promedio mensuales de los jefes de hogar

\begin{tabular}{cccc}
\hline ¿Cuáles son los ingresos mensuales promedio de la familia? & Jefe de hogar & Part. (\%) \\
\hline Menos de 1 SMLV & 12 & $36.36 \%$ \\
\hline 1 SMLV & 21 & $63.64 \%$ \\
\hline Más de 1 SMLV & 0 & $0.00 \%$ \\
Total & 33 & $100.00 \%$ \\
\hline
\end{tabular}

Fuente: Elaboración propia mediante encuesta (2018).

Según la tabla 16, la mayoría de los jefes de hogar (63.64\%), tienen ingresos de un salario mínimo, el 36.36\% gana menos de un salario mínimo. Esto muestra que todos necesitan generar más ingresos, por medio del fortalecimiento de las capacidades productivas y el emprendimiento.

Tabla 17.

Reporte de la vida crediticia del jefe de hogar en centrales de riesgo

\begin{tabular}{ccc} 
¿Se encuentra reportado en alguna central de riesgo? & Jefe de hogar & Part. $(\%)$ \\
SIFIN & 4 & $12.12 \%$ \\
\hline Datacredito & 12 & $36.36 \%$ \\
Covinoc & 0 & $0.00 \%$ \\
Otra & 0 & $0.00 \%$ \\
Ninguno & 17 & $51.52 \%$ \\
\hline Total & 33 & $100.00 \%$
\end{tabular}

Fuente: Elaboración propia mediante encuesta (2018).

En la tabla 17, se muestra que es alta la cantidad de jefes de hogares que se encuentran en estado de endeudamiento: $36.36 \%$ reportados en la central de riesgo Data Crédito, $12.12 \%$, reportados en la SIFIN, para un total de $48.48 \%$ de personas con una mala hoja de vida crediticia. Por otro lado, el $51.52 \%$ restante, que representa la mayoría de los jefes de hogar no presentan ningún tipo de deuda. Esto muestra que todos necesitan generar más ingresos, por medio del fortalecimiento de las capacidades productivas y el emprendimiento. 
Tabla 18.

Vinculación con entidades financieras de microcrédito

\begin{tabular}{ccc}
\hline $\begin{array}{c}\text { ¿Tiene vínculo con alguna entidad financiera de } \\
\text { microcrédito? }\end{array}$ & Jefe de hogar & Part. (\%) \\
\hline Bancamía & 12 & $36.36 \%$ \\
\hline Banco de oportunidades & 0 & $0.00 \%$ \\
\hline Banco privado & 0 & $0.00 \%$ \\
\hline Paga diario & 6 & $18.18 \%$ \\
\hline Otro & 0 & $0.00 \%$ \\
\hline Ninguna & 15 & $45.45 \%$ \\
\hline Total & 33 & $100.00 \%$ \\
\hline
\end{tabular}

Fuente: Elaboración propia mediante encuesta (2018).

La tabla 18, evidencia hay una buena cantidad de jefes de hogares que tienen microcréditos, como apalancamiento para sus negocios, siendo su vinculación con las siguientes entidades: Bancamía (36.36\%) y Paga diario (18.18\%), para un total de 54.54\%. El 45.45\% restante, no tienen ninguna vinculación con alguna entidad financiera. Esto muestra que estas personas requieren comprometerse y arriesgarse a financiar para capacitarse, emprender y generar más ingresos y negocios propios.

Tabla 19.

Vinculaciones con Fundaciones o Cooperativas ¿Se encuentra vinculado con alguna fundación o cooperativa? Jefe de hogar Part. (\%)

\begin{tabular}{ccc}
\hline Fundación Mario Santodomingo & 6 & $18.18 \%$ \\
\hline Fundación Mundo Mujer & 8 & $24.24 \%$ \\
\hline Famiempresa & 0 & $0.00 \%$ \\
\hline Actuar & 0 & $0.00 \%$ \\
\hline Otra & 0 & $0.00 \%$ \\
\hline Ninguna & 19 & $57.58 \%$ \\
\hline Total & 33 & $100.00 \%$ \\
\hline
\end{tabular}

Fuente: Elaboración propia mediante encuesta (2018).

La tabla 19, muestra que es buena la cantidad de jefes de hogares, que se encuentran vinculadas a Fundaciones o Cooperativas de desarrollo y de capacidad humana, siendo su vinculación con las siguientes entidades: Fundación Mario Santodomingo (18.18\%) y Fundación Mundo Mujer (24.24\%), para un total de 42.42\% de las personas. Por su parte, el 57.58\% restante, no tienen ninguna vinculación con alguna Fundación o Cooperativa. Esto muestra que estas personas necesitan motivarse, relacionarse y participar en estas organizaciones para generar mayores capacidades productivas, tener mayor apoyo social y económico, que le permitan mejorar sus condiciones de vida.

Tabla 20.

Estrategia de ahorro del jefe de hogar ¿Posee alguna estrategia de ahorro?

Personal Jefe de hogar Part. (\%)

Entidad 18 $54.55 \%$

Grupo de ahorro Ninguna Total

$0 \quad 0.00 \%$

$0 \quad 0.00 \%$

$15 \quad 45.45 \%$

$33 \quad 100.00 \%$

Fuente: Elaboración propia mediante encuesta (2018). 
Se puede observar que en la tabla 20 , el $54.55 \%$ de los jefes de hogar ahorran usando estrategias personales. El 45.45\% restante, no ahorra, ni utiliza ninguna estrategia de ahorro. Esto muestra que estas personas necesitan capacitarse más en administración y finanzas para que puedan aprender a tomar decisiones con base a estrategias ganadoras, que le permitan invertir mejor, tener recursos, activos y capital de trabajo que le generen mayor margen de utilidad y rentabilidad.

Tabla 21.

Percepción acerca del emprendimiento de un negocio

\begin{tabular}{ccr}
\hline ¿Le gustaría emprender un negocio? & Jefe de hogar & Part. (\%) \\
\hline $\mathrm{Si}$ & 24 & $72.73 \%$ \\
\hline $\mathrm{No}$ & 9 & $27.27 \%$ \\
\hline Total & 33 & $100.00 \%$ \\
\hline Fuente: Elaboración propia mediante encuesta $(2018)$. &
\end{tabular}

De acuerdo con la tabla 21, el 73\% de los jefes de hogar les gustaría emprender un negocio propio: Miscelánea $27.27 \%$, negocios en casa, tiendas para venta de productos de consumo de hogar, fritos y panaderías $27.27 \%$, internet $15.15 \%$, modistería $12.12 \%$, salas de belleza, barberías y peluquerías $6.06 \%$, otros $6.06 \%$ (zapatería y mantenimiento de equipos)

Con este análisis de investigación mixta, se busca que los beneficiarios del proyecto, se fortalezcan en programas de emprendimientos encaminadas a mejorar la calidad de vida, y se conviertan en productores de empleos auto sostenibles.

\section{Caracterización de las capacidades de CTI para la distribución y comercialización.}

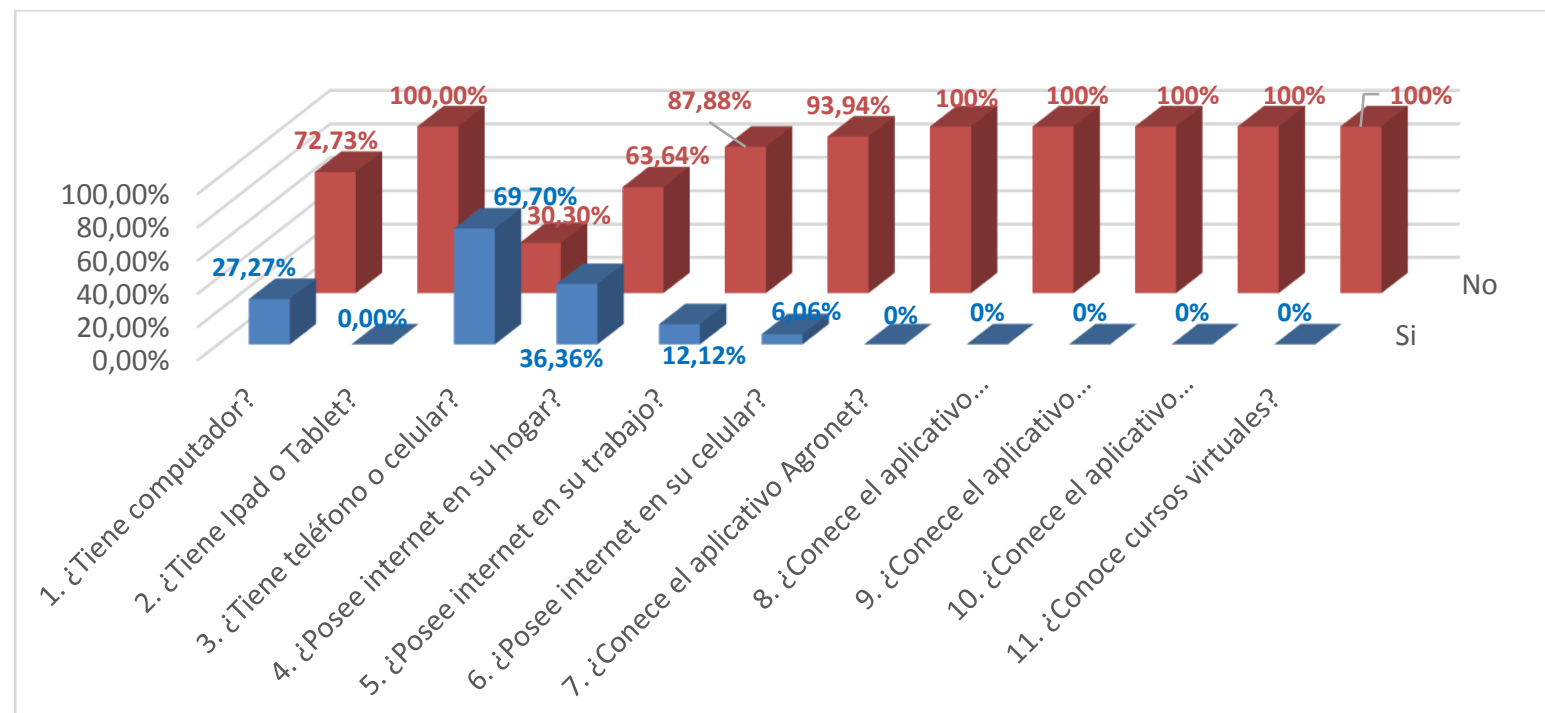

Figura 2. Equipamiento y capacidades de CTI para la distribución y comercialización por jefe de hogar del barrio la Ceiba de Baranoa, Atlántico.

Nota. Fuente: Elaboración propia con base al formato de caracterización de alfabetización digital de la Agencia de Desarrollo Rural, ADR de la FAO, la ONU y Ministerio de Agricultura y Desarrollo Rural, MADR (2018). 
Según la Figura 2, de un total de 33 jefes de hogar, pertenecientes al barrio La Ceiba, ninguno de ellos cuenta con tablets o Ipad, sólo 4 poseen conexión de internet en su trabajo y 2 en su celular; así mismo, ninguno conoce los aplicativos digitales de Ciencia Tecnología e Innovación-CTI para la distribución y comercialización: Agronet, Agronegocios, Celuagronet, Mipyme digital, ni otros cursos virtuales para aumentar sus ventas. Mientras que solo 9 tienen computador, 23 tienen celulares y 12 tienen conexión a internet en su hogar, como se evidencia desde la pregunta 22 hasta la 32 de la encuesta presentada en la gráfica 2, donde se observó el bajo nivel de alfabetización digital y de equipamiento de herramientas tecnológicas para la comercialización de sus capacidades y negocios de bienes y servicios, por lo cual en las preguntas abiertas de percepción de las tablas 9, 10, 11, 15 y 21, los hogares reconocen que necesitan apoyo de capacitación, incentivos tributarios y financieros, ofertas de inclusión y de conexión con el sector productivo en los programas de las instituciones o agencias del Estado, relacionadas con las áreas donde requieren capacitarse para tener mayores capacidades de conformar sus negocios y de generar ingresos sosteniblemente.

\section{Discusiones}

Los resultados presentados de la caracterización de las capacidades económicas en los aspectos: a) productivos y sociales, b) económicos y financieros y c) comerciales y tecnológicos, obtenidos por la Universidad de Oxford, mediante la teoría de las capacidades y funciones de Sen (1985) para la acumulación del conocimiento, son una buena alternativa para aplicar en Colombia por parte del gobierno nacional, departamental y distrital, dado el éxito que ha tenido en India, Perú y otros países tercermundistas, con situación de posconflicto; de la misma manera se están prestando servicios comunitarios directamente para impulsar a las familias por medio de los programas de salud, educación, capacitación gratuita y responsabilidad social del Sena, las Instituciones, Universidades y Organizaciones públicas y privadas, becas en educación como el programa de jóvenes en acción, Colciencias, apoyo económico y financiero para proyectos en actividades productivas, empleo, emprendimiento, comercialización y negocios mediante las TIC, que son incluyentes para la participación de las familias vulnerables en edad de trabajar. Sin embargo, la teoría de Sen (1995), no se puede cumplir en su totalidad, dado las condiciones que presenta el país, en donde el gobierno está mejorando las políticas públicas de sus instituciones, en cuanto a: la corrupción, al subsidio a familias, que no deben estar en el programa y a los que están, los cuales se han vuelto improductivos, no se esfuerzan para capacitarse y generar sus propios ingresos, "aprendiendo a pescar".

En cambio, otras teorías tradicionales como: las de las Necesidades Básicas Insatisfechas (NBI), las condiciones de vida (CV), la línea de pobreza (LP) y Mapas de Pobreza, realizadas por Max-Neef (1986), Rawls (1995), Feres y Mancero (2001), Davis (2003), Pérez (2005), Ospina y Giraldo (2005), Galvis y Meisel (2012) y Serafini (2015), no son multidimensionales, son menos optimistas y buscan disminuir el problema de la pobreza temporalmente, buscando resultados en la generación de ingresos lo más rápido posible, sin importar que se potencialicen las actividades y se generen ingresos por medio de subsidios. Estas teorías obedecieron a la preocupación que se vivió en su momento, con el afán de la ONU de llegar a las familias en pobreza extrema presentadas en los países menos desarrollados, actualmente, todos los autores coinciden en la medición de indicadores integrales o multidimensionales en todas las áreas. 
Teniendo en cuenta lo anterior, queda claro que las capacidades económicas y las funciones de las instituciones son herramientas que contribuyen benéficamente a reducir al problema de medición de los indicadores de pobreza multidimensional, de donde se deriva gran parte de la generación de valores económicos, originalmente representado en términos financieros y económicos como rentabilidad económica, progreso e innovación social y acumulación de conocimiento en la generación de sus propios recursos.

\section{Conclusiones}

Se puede concluir con el diagnóstico de la situación en los aspectos sociales, económicos, políticos, productivos, comerciales y tecnológicos, resultantes de la caracterización de las capacidades productivas en el barrio la Ceiba de Baranoa, con base en la teoría de las capacidades y funciones humanas de Amartya Sen 1995 que se registra una baja generación de ingresos en la población en edad de trabajar y los jefes de hogar encuestados en el barrio Baranoa, debido a que casi la mitad de los hogares no tiene cultura de ahorro, solo un poco más de la mitad, la maneja personalmente sin depositar en bancos, ni otros grupos de ahorro. También se registra que ha aumentado las deudas y gastos en casi la mitad de esta población, la cual aparece reportada por entidades financieras como Data Crédito y Sifin (Bancamía, Fundación Mundo Mujer y Pagadiarios), por lo cual requieren intervención de las instituciones del gobierno, fundaciones y cooperativas sociales, tanto nacionales como internacionales, en inclusión de programas de formación para el manejo de las finanzas y la generación de ingresos con proyectos productivos.

Sin embargo, existe la voluntad por parte de los jefes de hogar, de participar en los programas de generación de ingresos por instituciones del Estado, por lo cual mostraron en su mayoría estar interesados en capacitarse en ventas y administración de negocios, así mismo están interesados en emprender su propio negocio, relacionado con venta de misceláneas, venta de víveres en micro tiendas, venta de comida, panadería, modistería y salones de belleza o peluquerías y otros, tales como: calzado y mantenimiento y reparación de equipos. Por ende, el gobierno y las instituciones que velan por el bienestar de las localidades deben intervenir, fortalecer y potencializar las capacidades que tienen en este barrio vulnerable y abandonado para mejorar la situación económica del municipio de Soledad.

Por otro lado, la población en edad de trabajar presenta disponibilidad para realizar cualquier actividad y participar en los programas sociales y proyectos productivos, dado que en su mayoría están afiliados a un buen sistema de salud subsidiado, donde no han tenido ningún tipo de discapacidad ni tiene limitaciones graves. Sin embargo, existe en su mayoría un bajo nivel de educación en la mayoría de los jefes de hogar con estudios de secundaria incompleta, primaria y pocos analfabetas, por lo cual muchos están desempleados y trabajan como independientes, lo cual ha incidido en los problemas de desarrollo de la familia, tales como: separación de parejas y soltería, por su difícil situación económica. Además, ninguno de los participantes gana por encima del mínimo y muy pocos están pensionados, los cuales tienen servicios privados y formales de salud y educación, así como estabilidad económica en sus ingresos.

En la caracterización de la asociación de los 33 jefes de hogar del barrio la Ceiba de Baranoa Atlántico, así como en la población en edad de trabajar, se diagnosticó un muy bajo nivel educativo y de alfabetización digital, lo cual 
Luis Escorcia-Márquez, Johanna Padilla-Ortiz, Elkyn Lugo-Arias, Franklyn Ferrer-Manotas

muestra la necesidad de la comunidad en capacitarse en el manejo de aplicativos y páginas digitales de internet, por medio de computadores y móviles, dado que requieren de ayuda básica en el manejo de las Tics y mucha motivación y apoyo para aprender desde cero y puedan ser competitivos en el ámbito laboral y de los negocios, para obtener más conocimiento, experiencia, ofrecer sus productos y servicios, atender grandes pedidos, traer distribuidores y empresas que ayuden a comercializar, generar más ingresos y mejorar la calidad de vida integral de las familias tanto en salud, nutrición, educación, participación social y manejo de las finanzas personales.

De esta manera, es muy importante la multiplicación de estas campañas de promoción y capacitaciones a todas las personas del barrio en edad de trabajar para ampliar la visión y la capacidad de negociación de cada uno de sus miembros con el fin de crecer en su nicho de mercado y asegurar mayores ingresos en sus ventas. Dado que a medida que las personas del barrio en edad de trabajar participan en los proyectos institucionales van a articularse con el medio para potencializar sus capacidades productivas de manera sostenible, en torno a la ruta de la prosperidad de su hogar y en la formalización de sus negocios, emprendimiento y empleabilidad.

\section{Referencias}

Córdoba, R. (2006). Desarrollo humano y capacidades. Aplicaciones de la teoría de las capacidades de Amartya Sen a la educación. Revista española de pedagogía, 64(234), 365-380. Recuperado de https://revistadepedagogia.org/volume/lxiv/no-234/

Davis, R. (2003). Distribución del ingreso y pobreza en Chile. Entre el neoliberalismo y el crecimiento con equidad: tres décadas de política económica en Chile. Cepal: Santiago de Chile. Recuperado de https://repositorio.cepal.org/handle/11362/1782

Dworkin, R. (2018). Equality. In Steven, C. (Comp.). Political Philosophy in the Twenty-First Century (pp. 15-29). New York: Routledge. DOI: https://doi.org/10.4324/9780429498190

Evans, P. (2002). Collective capabilities, culture, and Amartya Sen's Development as Freedom. Studies in Comparative International Development, 37(2), 54-60. DOI: https://doi.org/10.1007/BF02686261

Feres, J. \& Mancero, X. (2001). El método de las necesidades básicas insatisfechas (NBI) y sus aplicaciones en América Latina. Santiago de Chiles: Cepal.

Galvis, L. \& Meisel, A. (2012). Convergencia y trampas espaciales de pobreza en Colombia. Documentos de trabajo sobre economía regional, (177). Cartagena, Colombia: Banco de la República.

Hatakka, M. (2013). The capability approach in ict4d research. (Doctoral theses, comprehensive summaries). Örebro universitet, Örebro, Suecia.

Henríquez-Fuentes, G., Lombana-Coy, J., González-Ariza, A., Higuera-Ojito, V., Landázury-Villalba, L., Rada-Llanos, J. \& Simancas-Trujillo, R. (2018). La gobernanza y su relación con la competitividad en una firma integrada a una cadena de suministro. Recuperado de http://bonga.unisimon.edu.co/handle/123456789/2086. 
Landázury, L., Basso, M., Neugovsen, G., Mercant, S., Jaafar, H. \& Ruz, A. (2018). Toma de decisiones y sus repercusiones sobre costos transaccionales en las empresas. Revista espacios, 39(24). Recuperado de http://bonga.unisimon.edu.co/handle/123456789/2124

Landazury, L., Jaafar, H., Cristofani, M. y Canales, R. (2018). Innovación y modelos de gerencia: su reflexión transformadora desde lo humano y el conocimiento. Revista espacios, 39(13). Recuperado de https://www.revistaespacios.com/a18v39n13/a18v39n13p20.pdf

Landázury, L., Lechuga, J., Ferrer, F. \& Prieto, R. (2017). Innovación como eje transversal en los negocios internacionales. En Ziritt, G., Taboada, R., Prieto, F., Jiménez, L. \& Barbosa, J. (comp.). Producción de Conocimiento desde el Campo de las Ciencias Económicas, Administrativas y Contables. Barranquilla: Universidad Simón Bolívar.

Lugo, E., Landázury, L., Ferrer, F., Roncallo, A., Lugo, J., Jaafar, H. \& Ovallos, D. (2018). Productividad, competitividad y creación de valor del sector agroexportador: un enfoque de los costos, la responsabilidad social y la gestión ambiental en la Región Caribe. Barranquilla: Ediciones Universidad Simón Bolívar.

Lugo, E., Sáenz, J. \& Lugo, J. (2018). La productividad como factor de competitividad de las exportaciones de aceite de palma del departamento del Magdalena Colombia, 2007-2015. Revista ciencia, saber y libertad, 13(1):145:163. DOI: https://doi.org/10.18041/2382-3240/saber.2018v13n1.2088

Lugo, J. \& Lugo, E. (2018). Beneficios socio ambientales por potabilización del agua en los pueblos palafíticos de la Ciénaga Grande de Santa Marta-Colombia. Revista U.D.C.A, actualidad y divulgación científica 21(1): 259264. DOI: https://doi.org/10.31910/rudca.v21.n1.2018.685

Max-Neef, M., Elizalde, A., Hopenhayn, M. (1986). Desarrollo a escala humana: una opción para el futuro. Santiago de Chile: Biblioteca CF + S.

Méndez, J. (2002). Determinantes de la pobreza en Colombia: años recientes. United Nations Publications.

Michalos, A. (2017). What did Stiglitz, Sen and Fitoussi get right and what did they get wrong?. In Michalos, A. (Comp.). Development of Quality of Life Theory and its Instruments (pp. 287-300). Spain: Springer.

Nussbaum, M. (2002). Las mujeres y el desarrollo humano. Barcelona, Herder.

Nussbaum, M. (2003). Capabilities as fundamental entitlements: Sen and social justice. Feminist economics, 9(2-3), 33-59.

Nussbaum, M. (2006). Frontiers of Justice. Disability, Nationality, Species Membership. United States of America, Harvard University Press. 
Nussbaum, M. (2011). Creating Capabilities. The Human Development Approach. United States of America: Harvard University Press.

Ospina, R. \& Giraldo, O. (2005). Aproximación a los conceptos de pobreza y distribución del ingreso. Semestre económico, 8(15), 47-61. Recuperado de https://www.redalyc.org/articulo.oa?id=165013659003

Pérez, G. (2005). Dimensión espacial de la pobreza en Colombia. Documentos de trabajo sobre economía regional, 54(1). Cartagena, Colombia: Banco de la República.

Petrucci, A., Salvati, N. \& Seghiari, C. (2003). The application of a spatial regression model to the análisis and mapping poverty. Environment and Natural Resources Service No 7. Sustainable Development Department. FAO: Roma.

Rawls, J. (1995). Teoría de la Justicia. México: Fondo de Cultura Económica.

Robeyns, I. (2005). The Capability Approach: a theoretical survey. Journal of Human Development, 6(1), 93-114. DOI: https://doi.org/10.1080/146498805200034266

Sánchez, F. \& Núñez, J. (1999). Descentralización, pobreza y acceso a los servicios sociales ¿Quién se benefició del gasto público social? Cepal: Santiago de Chile. Recuperado de https://www.cepal.org/es/publicaciones/7595descentralizacion-pobreza-acceso-servicios-sociales-quien-se-beneficio-gasto

Sen, A. (1985). Commodities and Capabilities. Amsterdam: North-Holland.

Sen, A. (1992). Inequality Reexamined. (Traducción al español de Bravo \& Schwartz). United States: Oxford University Press.

Sen, A. (1995). Nuevo Examen de la Desigualdad. Madrid: Alianza Editorial.

Sen, A. (2018). Collective choice and social welfare. United States: Harvard University Press.

Serafini, V. (2015). Pobreza, oportunidades económicas desiguales y género. Asunción: ONU Mujeres, PNUD.

Stiglitz, J., Sen, A. \& Fitoussi, J. (2010). Mismeasuring our lives: Why GDP doesn't add up. Nueva York: The New Press.

Universidad Minuto de Dios. (2016). Estructura organizacional de la Proyección Social. Diseño de un modelo de intervención Psicosocial y económico para la población vulnerable del municipio de Baranoa: Barrio La Ceiba y el municipio de Soledad: Barrio Ciudad Bonita. Recuperado de https://n9.cl/7xz9 
Luis Escorcia-Márquez, Johanna Padilla-Ortiz, Elkyn Lugo-Arias, Franklyn Ferrer-Manotas

Urquijo, M. (2014). La teoría de las capacidades en Amartya Sen. Revista Edetania, (46), 63-80. Recuperado de https://dialnet.unirioja.es/servlet/articulo?codigo $=5010857$

Walker, M. \& Unterhalter, E. (2007). Amartya Sen's Capability Approach and Social Justice in Education. Editorial: Palgrave Macmillan

Wells, T. (2012). Sen's Capability Approach. Internet Encyclopedia of Philosophy. Recuperado de https://www.iep.utm.edu/sen-cap/

Yunus, M., Jolis, A. \& Morshed, L. (2006). El banquero de los pobres: los microcréditos y la batalla contra la pobreza en el mundo. Barcelona: Paidós. 